

Check for updates

\title{
Stress Analysis of a Burch-Schneider Cage in an Acetabular Bone Defect: A Case Study
}

Plessers $\mathrm{K}^{1}$, Mau $\mathrm{H}^{2}$

\begin{abstract}
Burch-Schneider cages are often used for the treatment of acetabular bone defects. In several clinical studies these cages have shown good mid- to long-term results. However, a higher failure rate has been reported in large Paprosky IIIB defects compared with smaller Paprosky II-IIIA defects. This study aims to investigate the effect of cage support on cage failure by means of finite element analysis. The Von Mises stresses in both the implant and the bone are analyzed for a Burch-Schneider cage used in the following scenarios: (1) a large acetabular bone defect, (2) a small acetabular bone defect and (3) a large acetabular bone defect in combination with a reinforcement plate. The results show that implant and bone stresses are higher in the large defect (99th percentile of 146.6 and 73.5 MPa respectively) than in the small defect (99th percentile of 43.9 and $47.9 \mathrm{MPa}$ respectively). Adding a reinforcement plate to posteriorly support the cage decreases the stresses but not fully compensates for the missing bone support (99th percentile of 93.1 and 55.3 MPa respectively). Since high stresses cause an increased risk for fatigue failure and implant loosening, sufficient implant support is required to reduce the risk of cage failure.
\end{abstract}

Keywords: Burch-Schneider cage, finite element analysis, Von Mises stresses, acetabular defect, bone support Level of Evidence: AAOS Therapeutic Level IV

\section{Introduction}

Implant fracture and aseptic loosening are common failure modes in hip arthroplasty. They can be caused by screw breakage, bone resorption, fractures and infections $[1,2]$. Besides pain, implant failure often leads to an enlargement of the bone defect. To restore the function of the hip, a complex revision surgery is required. Removing the old components and reconstructing the enlarged bone defect may involve a high complication risk for the patient and a high financial cost for the medical institution. Therefore, a durable surgical treatment should be selected for
1 Katrien Plessers, MSc,

Materialise N.V., Leuven, Belgium

(Direct reprint requests to Katrien Plessers)

2 Dr. Hans Mau

Helios Endo-Klinik Hamburg, Hamburg, Germany

(C) 2016 Plessers, Mau. All rights reserved.

Authors retain copyright and grant the journal right of first publication with the work. Reconstructive Review follows the Creative Commons Attribution-NonCommercial

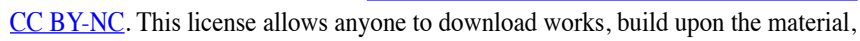
and share them with others for non-commercial purposes as long as they credit the senior author, Reconstructive Review, and the Joint Implant Surgery \& Research Foundation (JISRF). An example credit would be: "Courtesy of (senior author's name), Reconstructive Review, JISRF, Chagrin Falls, Ohio". 
each patient from the beginning.

In the last 30 years, the Burch-Schneider cage has been widely used to reconstruct acetabular bone defects [2]. This type of anti-protrusio cage is often used to bridge bone defects with a perforation of the medial wall. The BurchSchneider cage is made of pure titanium (Protasul Ti) [1] and consists of a hemispherical shell with an inferior and a superior flange. The inferior flange is impacted into a prepared slot in the ischial bone while the superior flange is fixed with screws to the iliac bone. The impaction into the ischium assures a rotationally stable implant. For a better bone contact of the implant, the flanges of the cage can be preoperatively bent according to the anatomical shape of the bone. After insertion, a cup insert is cemented into the hemispherical shell of the cage $[1,2]$.

The Burch-Schneider cage has shown good results at mid- and long-term in the treatment of acetabular bone defects. Regis et al. [3] reported a survival rate of $80 \%$ $(52 / 65)$ for an average of 14.9 years follow-up. Sembrano et al. [4] demonstrated a 5-year loosening-free survival rate of $80.7 \%$ for 72 cage reconstructions. Lamo-Espinosa et al. [5] showed a 79\% survival rate at 10 years after implantation in 16 hips. The study of Hsu et al. [6] indicated a 5-year survival rate of $76 \%$ in 31 cage reconstructions. However, these studies do not separately report results for defects of different Paprosky classification [7]. Other clinical studies on the contrary show a higher failure rate in bone defects of class Paprosky IIIB compared to smaller bone defects: Perka et al. [8] found a direct correlation between Burch-Schneider cage migration and posterior column defects in increasing Paprosky stages. Udomkiat et al. [9] determined that Burch-Schneider cage migration also correlated with the amount of superior support from the ilium. Paprosky et al. [10] reported a failure of 7/11 (63.6\%) ilioischial acetabular systems in type IIIB defects, because insufficient host bone was available to support a cage.

To improve cage stability in large and uncontained bone defects, surgeons may use a defect-filling structure that provides additional support to a Burch-Schneider cage. Structural allografts are commonly used in defects with severely degraded acetabular bone stock $[6,11,12,13]$. A disadvantage of allografts is the complex surgical procedure they require to shape and fit the allograft to the bone defect. Moreover, without sufficient host bone, allografts may degrade or collapse due to a change in mechanical stimuli $[3,9,10,13]$. Another commonly used defect-filling structure is trabecular metal $[13,14]$. Trabecular metal augments are made of porous tantalum and are available in different shapes. However, additional reaming of the bone defect may be required to achieve a good fit of the augment. As an alternative to the Burch-Schneider cage and the re- quired support structures, patient-specific implants are increasingly used for the treatment of large acetabular bone defects. Patient-specific implants have already proven their added-value in short-term patient outcome [15]. These implants can be designed as a cup with two or three flanges and a porous defect-filling scaffold. The personalized fit between the scaffold and the bone provides a large contact area for enhanced support and stability of the implant [16]. However, patient-specific implants are more expensive than standard available implants and are therefore limited to patients with few other options.

In general, implant stresses are known to have an impact on the implant's fatigue lifetime [17]. If the Von Mises stresses in an implant exceed the fatigue limit, the implant has an increased risk for breakage or fatigue failure after consecutive loading and unloading. Bone stresses on the other hand play a role in the bone remodeling process which subsequently may induce implant loosening $[18,19]$. If the stress distribution in the bone deviates from the natural and healthy mechanical conditions, the bone has an increased risk for bone degradation $[20,21]$. To evaluate implant designs and analyze implant performance under different loading conditions, finite element (FE) methods have been widely used $[22,23,24]$.

The goal of this study is to analyze if the effect of BurchSchneider cage support on the implant and bone stresses can be demonstrated by means of finite element analysis (FEA). Therefore, four scenarios are evaluated: first of all, the cage behavior is analyzed in a clinical case of a patient having a large acetabular bone defect of type Paprosky IIIB. Secondly, the Burch-Schneider cage is evaluated in a smaller acetabular bone defect which provides more bone support to the cage. Thirdly, a scenario is analyzed in which the large acetabular bone defect is treated with a cage in combination with a reinforcement plate. Surgeons may add such a reinforcement plate to provide some additional support to the cage. Reinforcement plates are mainly used in large uncontained bone defects or dissociations in order to avoid cage instability $[11,12,25]$. Finally, a healthy pelvis is evaluated as a reference model.

\section{Materials and methods}

The following finite element models were created in Abaqus (Simulia, 3DS, Paris, France):

1. Large defect model: a Burch-Schneider cage used in a large acetabular bone defect. The large bone defect only provides support to the flange regions of the cage and to a small part of the cup.

2. Small defect model: a Burch-Schneider cage used 
in a small acetabular bone defect. Additional bone behind the cup enlarges the contact region between cage and bone.

3. Plate model: a Burch-Schneider cage used in a large bone defect, but in combination with a reinforcement plate. Except for the plate, this model is exactly the same as the large defect model. The added reinforcement plate provides support to the posterior rim of the cage.

4. Healthy model: a model of a healthy hemi-pelvis that acts as a reference model.

\section{Geometry models}

The bone geometry of a 60 -year old female patient ( 70 $\mathrm{kg}$ ) was used in the finite element models. The left hemipelvis of the patient was dissociated and the acetabulum was completely degraded [26]. The defect was classified as Paprosky IIIB by the patient's surgeon. The 3D geometry of the bone (Figure 1a) was segmented from the CT scan by the image processing software Mimics (Materialise N.V., Leuven, Belgium) [27]. In addition to the severely defected hemipelvis, a bone model with a small defect was artificially created. The artificial bone model was generated by manually decreasing the bone defect of the patient (Figure 1b) in the 3d modeling software 3-matic (Materialise N.V., Leuven, Belgium). The acetabular cup was partially reconstructed by focusing on the geometry of a healthy pelvis. Furthermore, a healthy bone model was generated by reconstructing the severe bone defect of the patient with a statistical shape model (Figure 1c). Thus, three different bone models were developed, including a hemi pelvis with a large defect, a hemi-pelvis with a small defect and a hemi-pelvis with no defect.

In order to model the implant geometry, a Burch-Schnei-

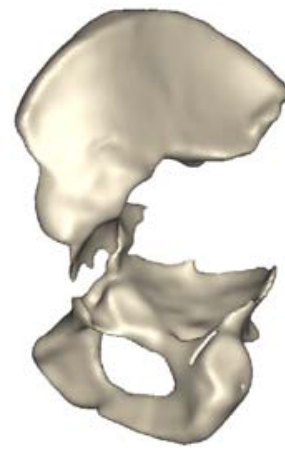

(a)

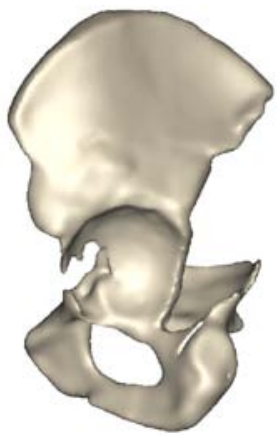

(b)

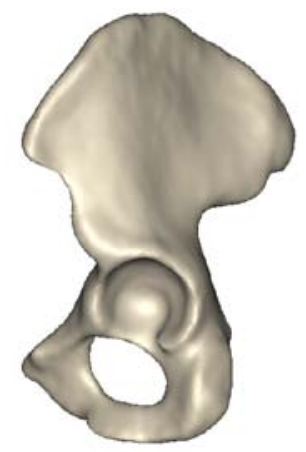

(c)
Figure 1. (a) Hemi-pelvis of a patient with a Paprosky IIIB defect, (b) hemi-pelvis with an artificial defect derived from (a), and (c) healthy hemi-pelvis which is the reconstruction of $(a)$.

der cage of size $62 \mathrm{~mm}$ (Zimmer Biomet, Warsaw, Indiana) and a $3.5 \mathrm{~mm}$ dynamic compression plate (DePuy Synthes,
Warsaw, Indiana) were optically scanned and processed by the software program 3-matic (Materialise N.V., Leuven, Belgium). The position of the Burch-Schneider cage on the bone was indicated by an experienced surgeon and was the same in the three models. The reinforcement plate was only present in the plate model. The surgeon positioned the plate on the posterior bone so that it provided support to the posterior rim of the cage (Figure 2).



Figure 2. Position of the BurchSchneider cage and the reinforcement plate in the large defect model

\section{FE model}

The finite element modeling approach was based on studies in literature [28,29,30,31]. As in these studies, a linear and static approach was applied. The bone geometries were meshed with linear tetrahedral elements of $2 \mathrm{~mm}$ average edge length [28]. Each bone was modeled as a trabecular inner region (volume elements) with an E-modulus of $300 \mathrm{MPa}$ and a $1 \mathrm{~mm}$ cortical outer shell (shell elements) with an E-modulus of $17 \mathrm{GPa}$ [28]. The Burch-Schneider cage and the reinforcement plate were both modeled with linear tetrahedral elements of $1 \mathrm{~mm}$ average edge length and an E-modulus of 110GPa (titanium). The contacts between plate and bone, and cage and bone were modeled as bonding contacts. In bonded contacts, no displacements are allowed in axial and tangential directions. This way of modeling the implant-bone interface is a simplification of the actual screw connection between bone and both implants. The screws on the cage and the plate only partially inhibit the tangential motions, mostly in the regions of the screws. However, since a compressive force is acting on the cage, the tangential motions will be limited and the connection can be approximated by a bonding contact.

All models were rigidly fixed at the pubic symphysis and the sacroiliac joint of the hemi-pelvis, indicated in Figure 3 $[28,32,33]$. According to Bergmann et al. [34], an average hip joint experiences a peak load of $238 \%$ of body weight during normal walking. Therefore in all models a static load of

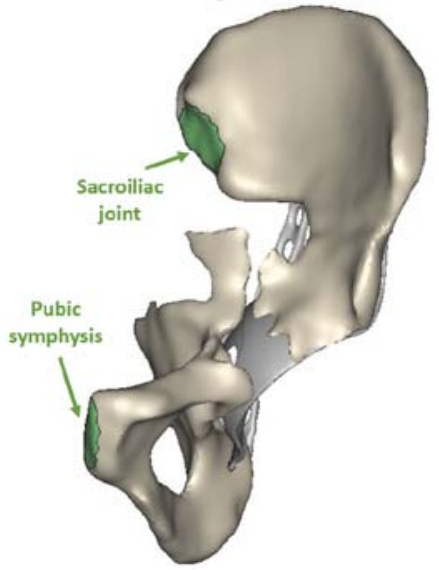

Figure 3. Illustration of the fixed boundary regions 
$1800 \mathrm{~N}$ ( $\approx 2.5$ times body weight) was applied in the cup of the Burch-Schneider cage or in the acetabulum. The force was applied along the vector $(0.13,-0.12,0.98)$ in the pelvic coordinate system, which is in accordance with Bergmann et al. [34]

\section{FE stress analysis}

To evaluate the failure risk of the Burch-Schneider cage, the Von Mises stresses in the cage and in the cortex were calculated. The fatigue strength of pure titanium for $10^{\wedge} 7$ cycles is $350-450 \mathrm{MPa}[35,36,37,38]$, depending on the precise material characteristics and the production process of the implant. If the maximum Von Mises stress in the cage approximates this fatigue stress, the cage has a higher risk for fatigue failure. The risk for bone degradation on the other hand increases when the stress distribution in the cortex deviates from the natural and healthy mechanical conditions $[20,21]$. The Von Mises stresses in the healthy model were analyzed as a reference value.

\section{Results}

Figures 4 and 5 show the stress distributions by color plots and cumulative graphs for the four models. The Von Mises stresses are highest in the large defect model and lowest in the small defect model. To exclude high peak stresses caused by modeling errors, the 99th percentile value is reported for each model instead of the maximum value (Table 1). The large defect model results in a 99th percentile value of $146.6 \mathrm{MPa}$ in the cage and $73.5 \mathrm{MPa}$ in the cortex. The small defect model shows a 99th percentile value of $43.9 \mathrm{MPa}$ and $47.9 \mathrm{MPa}$ in the cage and the cortex respectively. Adding a reinforcement plate to the large acetabular bone defect in the plate model gives a 99th percentile value of 93.1 MPa in the cage and 55.3 MPa in the cortex. In the healthy model, only the cortex stresses are assessed leading to a 99th percentile value of $37.6 \mathrm{MPa}$. The latter is used as a reference value for the stress distribution in a healthy pelvis.

The stresses in the cage are highest in the rim and the

Table 1: 99th percentile values of the Von Mises stress in the cage and the cortex

\begin{tabular}{|c|c|c|}
\hline Model & $\begin{array}{c}\text { Cage stresses } \\
{[\mathbf{M P a}]}\end{array}$ & $\begin{array}{c}\text { Cortex stresses } \\
{[\mathbf{M P a}]}\end{array}$ \\
\hline Large defect & 146.6 & 73.5 \\
\hline Small defect & 43.9 & 47.9 \\
\hline Plate & 93.1 & 55.3 \\
\hline Healthy & - & 37.6 \\
\hline
\end{tabular}

(a)

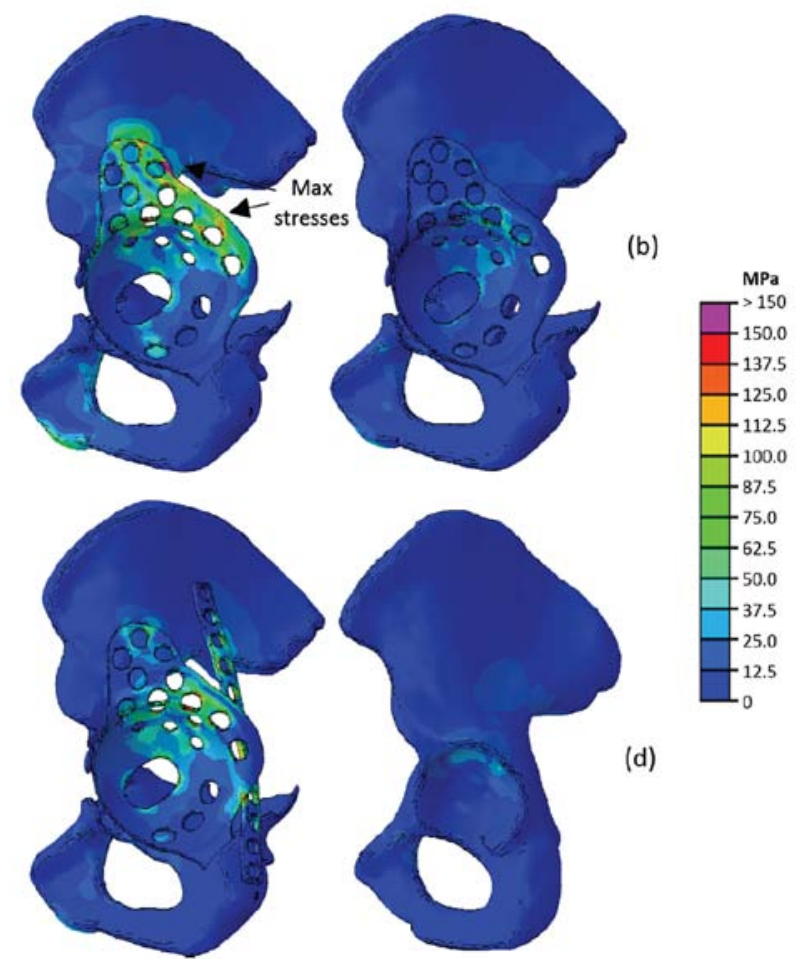

Figure 4: Stress distribution plots of (a) a Burch-Schneider cage in a large acetabular bone defect, (b) a Burch-Schneider cage in a small acetabular bone defect, (c) a Burch-Schneider cage in a large acetabular bone defect in combination with a reinforcement plate and (d) a healthy pelvis

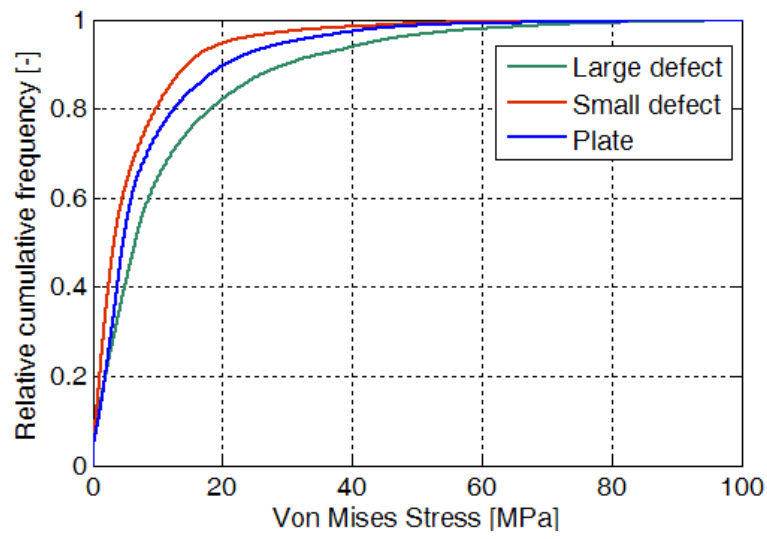

(a)



(b)

Figure 5: Cumulative stress distribution plots in (a) the cage and (b) the cortex. 
iliac flange (indicated on Figure 4a). However, for all models the 99th percentile stresses in the cage are still within the fatigue limit of pure titanium. The large defect model shows the highest stress values. The 99th percentile cage stress in the large defect model is respectively $234 \%$ higher than the small defect model, and $58 \%$ higher than the plate model. Adding a reinforcement plate in the large bone defect reduces the 99th percentile stress in the cage by $37 \%$. Furthermore, the cortex stresses in the large defect model, the small defect model and the plate model show a deviation of respectively $95 \%, 27 \%$ and $47 \%$ with respect to the healthy scenario. The small defect model thus most closely approaches the healthy pelvis.

\section{Discussion}

Selecting a suitable treatment option for each patient is essential in order to avoid implant failure in hip arthroplasty. Clinical studies have reported that support beneath a Burch-Schneider cage has a large effect on the implant survival rate $[6,8,9,10]$. This study investigated if the effect of Burch-Schneider cage support on the implant and bone stresses can be demonstrated by means of finite element analysis.

The FEA results indicated that support beneath the cage has a large effect on the stresses in the implant and the bone. A large support area beneath the cage limited the presence of peak stresses and resulted in a more equal stress distribution. The large bone defect in this study only provided support to the flange regions of the cage and to a small part of the cup. This limited support resulted in high stresses in both the implant and the bone. In the small defect model, the additional bone behind the cup enlarged the support region and so improved the force distribution. As a consequence, stress concentrations and peak stresses were reduced. In the plate model, little host bone was available to support the cage, but an extra reinforcement plate provided support to the posterior rim of the cage. This extra support protected the cage from excessive bending moment and reduced the high stress concentrations that were present in the large defect model. However, the additional support of the reinforcement plate did not fully compensate for the missing bone support.

Although the Von Mises stresses in the cage were below the fatigue limit for all models, some additional aspects should be considered. First of all, the current FE analyses only calculated the peak stresses during normal walking with a patient weight of $70 \mathrm{~kg}$. For heavier patients and more demanding activities, like running or stumbling, the loads on the pelvis can be 3-6 times as high [34]. Since this is a linear analysis, the stresses in the implant and the bone would increase in proportion with the applied force. Secondly, the fatigue performance of the cage also worsens when the surgeon preoperatively bends the flanges of the cage to fit the shape of the patient's bone. Plastic deformation in the flanges decreases the fatigue strength and therefore additionally reduces the fatigue lifetime of the cage. So despite the acceptable Von Mises stresses, the simplicity of the current FE models makes it difficult to make conclusions about the actual fatigue lifetime. However, the FE analysis does indicate that the large defect model has a higher risk for fatigue failure than the other two models. A cage should thus be sufficiently supported in order to avoid fatigue failure.

Besides implant stresses, cage failure was investigated by evaluating bone stresses. A change in mechanical loading of the bone with respect to the healthy situation affects the bone remodeling process $[20,21]$. The bone surrounding the implant may degrade which subsequently leads to implant loosening. The deviation from the healthy scenario was largest for the large defect model. This indicates that the large defect model has the highest risk for bone degradation and implant loosening.

The outcome of this study corresponds with the findings in literature: sufficient support should be available for long-term stability of a Burch-Schneider cage $[6,8,9,10]$. These findings were based on the relatively high failure rates of cages in Paprosky IIIB defects compared to smaller defects. Therefore, in large and uncontained bone defects defect-filling structures such as bone allografts and trabecular metal can be used to improve the cage support. Besides, patient-specific implants have already shown successful results in the treatment of large and challenging bone defects and should be considered as a potential alternative to the Burch-Schneider cage.

The main limitation of the current study is that it only involves a numerical analysis. Although some assumptions have been made regarding material properties, loading conditions and boundary conditions, the finite element results are in good agreement with other FEA results in literature. Philips et al. [30] describes an FE model of a healthy pelvis with loading and boundary conditions similar to the ones used in our models. The authors observe high stress concentrations at the superior rim of the acetabulum and towards the sacro-iliac joint with a maximum around 70MPa. Anderson et al. [28] reported a subject-specific finite element analysis of a healthy pelvis resulting in Von Mises stresses in the range of $0-44 \mathrm{MPa}$ for the cortex. The Von Mises stress distribution in our healthy model agrees with the results described in these previous stud- 
ies. Future finite element studies are required to investigate the effect of patient-specific modeling parameters and to improve the current modeling approach, specifically the boundary conditions, loading scenario and material properties. Moreover, experimental tests should be performed as a validation of the final simulation results.

\section{Conclusion}

The FEA results showed that support beneath the Burch-Schneider cage has a large effect on the stresses in both the implant and the bone. A large support area beneath the cage limits the presence of peak stresses and results in a more equal stress distribution. Specifically, in a large Paprosky IIIB acetabular bone defect the stresses in implant and bone were higher than in a small bone defect. Adding a reinforcement plate to posteriorly support the cage in the large bone defect decreased the stresses but did not fully compensate for the missing bone support. The high implant and bone stresses that occurred due to limited support regions caused an increased risk for fatigue failure and implant loosening. Hence to reduce the risk of implant failure, the surgeon should strive for optimal support.

\section{Disclosure}

The authors declare that there is no conflict of interest regarding the publication of this paper. For full disclosures refer to last page of this journal.

\section{References}

1. J. Gallo and A. V. Florchutz, "Burch-Schneider cage fracture: a case report," Biomed. Pap.PALACKY Univ. OLOMOUC, vol. 149, no. 2, p. 281, 2005

2. Y. Kosashvili, O. Safir, D. Backstein, D. Lakstein, and A. E. Gross, "Salvage of Failed Acetabular Cages by Nonbuttressed Trabecular Metal Cups," Clin. Orthop. Relat. Res., vol. 468 no. 2, pp. 466-471, Feb. 2010.

3. D. Regis, A. Sandri, and I. Bonetti, "Acetabular Reconstruction with the Burch-Schneider Antiprotrusio Cage and Bulk Allografts: Minimum 10-Year Follow-Up Results,” BioMed Res. Int., vol. 2014, pp. 1-9, 2014.

4. J. N. Sembrano and E. Y. Cheng, "Acetabular Cage Survival and Analysis of Factors Related to Failure," Clin. Orthop., vol. 466, no. 7, pp. 1657-65, Jul. 2008

5. J. Lamo-Espinosa, J. D. Clemente, P. Díaz-Rada, J. Pons-Villanueva, and J. R. Valentí-Nín, "The Burch-Schneider antiprotrusio cage: medium follow-up results," Musculoskelet. Surg., vol. 97, no. 1, pp. 31-37, Dec. 2012.

6. C.-C. Hsu, C.-H. Hsu, S.-H. Yen, and J.-W. Wang, "Use of the Burch-Schneider cage and structural allografts in complex acetabular deficiency: 3- to 10-year follow up," Kaohsiung J. Med. Sci., 2015

7. W. G. Paprosky, P. G. Perona, and J. M. Lawrence, "Acetabular defect classification and surgical reconstruction in revision arthroplasty: A 6-year follow-up evaluation," J. Arthroplasty, vol. 9, no. 1, pp. 33-44, Feb. 1994.

8. C. Perka and R. Ludwig, "Reconstruction of segmental defects during revision procedures of the acetabulum with the Burch-Schneider anti-protrusio cage," J. Arthroplasty, vol. 16, no. 5 pp. 568-574, Aug. 2001

9. P. Udomkiat, L. D. Dorr, Y.-Y. Won, D. Longjohn, and Z. Wan, "Technical factors for success with metal ring acetabular reconstruction," J. Arthroplasty, vol. 16, no. 8, pp. 961-969, Dec. 2001.

10. W. G. Paprosky, S. S. Sporer, and B. P. Murphy, “Addressing Severe Bone Deficiency: What a Cage Will Not Do,” J. Arthroplasty, vol. 22, no. 4, Supplement, pp. 111-115, Jun. 2007.

11. D. J. Berry, D. G. Lewallen, A. D. Hanssen, and M. E. Cabanela, "Pelvic Discontinuity in Revision Total Hip Arthroplasty*," J Bone Jt. Surg Am, vol. 81, no. 12, pp. 1692-1702, Dec. 1999.
12. J. B. Stiehl, R. Saluja, and T. Diener, "Reconstruction of major column defects and pelvic discontinuity in revision total hip arthroplasty," J. Arthroplasty, vol. 15, no. 7, pp. 849-857, oktober 2000

13. T. J. Mäkinen, S. G. Fichman, E. Watts, P. R. T. Kuzyk, O. A. Safir, and A. E. Gross, "The role of cages in the management of severe acetabular bone defects at revision arthroplasty," Bone Jt. J, vol. 98-B, no. 1 Supple A, pp. 73-77, Jan. 2016

14. S. M. Sporer and W. G. Paprosky, "The Use of a Trabecular Metal Acetabular Component and Trabecular Metal Augment for Severe Acetabular Defects," J. Arthroplasty, vol. 21, no. 6 , Supplement, pp. 83-86, Sep. 2006.

15. M. Baauw, G. G. van Hellemondt, M. L. van Hooff, and M. Spruit, "The accuracy of positioning of a custom-made implant within a large acetabular defect at revision arthroplasty of the hip," Bone Jt. J., vol. 97, no. 6, pp. 780-785, 2015

16. D. K. DeBoer, M. J. Christie, M. F. Brinson, and J. C. Morrison, "Revision Total Hip Arthroplasty for Pelvic Discontinuity," J. Bone Jt. Surg., vol. 89, no. 4, pp. 835-840, Apr. 2007.

17. T. Villa, F. Migliavacca, D. Gastaldi, M. Colombo, and R. Pietrabissa, "Contact stresses and fatigue life in a knee prosthesis: comparison between in vitro measurements and computational simulations," J. Biomech., vol. 37, no. 1, pp. 45-53, Jan. 2004

18. D. Lacroix, A. Chateau, M.-P. Ginebra, and J. A. Planell, "Micro-finite element models of bone tissue-engineering scaffolds," Biomaterials, vol. 27, no. 30, pp. 5326-5334, Oct. 2006.

19. C. Boyle and I. Y. Kim, "Comparison of different hip prosthesis shapes considering micro-level bone remodeling and stress-shielding criteria using three-dimensional design space topology optimization," J. Biomech., vol. 44, no. 9, pp. 1722-1728, Jun. 2011.

20. F. Schmidutz, Y. Agarwal, P. E. Müller, B. Gueorguiev, R. G. Richards, and C. M. Sprecher, "Stress-shielding induced bone remodeling in cementless shoulder resurfacing arthroplasty: a finite element analysis and in vivo results," J. Biomech., vol. 47, no. 14, pp. 3509-3516, Nov. 2014.

21. J. H. Kuiper and R. Huiskes, "The predictive value of stress shielding for quantification of adaptive bone resorption around hip replacements," J. Biomech. Eng., vol. 119, no. 3, pp. 228-231, 1997.

22. M. Rabbani and H. Saidpour, "Stress Analysis of a Total Hip Replacement Subjected to Realistic Loading Conditions," J Robot Mech Eng Resr, vol. 1, no. 1, pp. 18-23, 2015.

23. Y. Hirata, Y. Inaba, N. Kobayashi, H. Ike, H. Fujimaki, and T. Saito, "Comparison of Mechanical Stress and Change in Bone Mineral Density Between Two Types of Femoral Implant Using Finite Element Analysis," J. Arthroplasty, vol. 28, no. 10, pp. 1731-1735, Dec. 2013.

24. J. M. F. K. Takahashi, A. C. Dayrell, R. L. X. Consani, M. A. de A. Nóbilo, G. E. P. Henriques, and M. F. Mesquita, "Stress Evaluation of Implant-Abutment Connections Under Different Loading Conditions: A 3D Finite Element Study," J. Oral Implantol., vol. 41, no. 2, pp. 133-137, 2015.

25. I. Ilyas, H. A. Alrumaih, S. Kashif, S. A. Rabbani, and A. H. Faqihi, "Revision of Type III and Type IVB Acetabular Defects With Burch-Schneider Anti-Protrusio Cages," J. Arthroplasty, vol. 30, no. 2, pp. 259-264, Feb. 2015

26. F. Gelaude, T. Clijmans, and H. Delport, "Quantitative Computerized Assessment of the Degree of Acetabular Bone Deficiency: Total radial Acetabular Bone Loss (TrABL)," Adv. Orthop., vol. 2011, p. e494382, Oct. 2011

27. F. Gelaude, T. Clijmans, P. L. Broos, B. Lauwers, and J. V. Sloten, "Computer-aided planning of reconstructive surgery of the innominate bone: Automated correction proposals," Comput. Aided Surg., vol. 12, no. 5, pp. 286-294, Jan. 2007.

28. A. E. Anderson, C. L. Peters, B. D. Tuttle, and J. A. Weiss, "Subject-Specific Finite Element Model of the Pelvis: Development, Validation and Sensitivity Studies," J. Biomech. Eng., vol. 127, no. 3, pp. 364-373, Feb. 2005.

29. M. Dalstra and R. Huiskes, "Load transfer across the pelvic bone," J. Biomech., vol. 28, no. 6, pp. 715-724, Jun. 1995

30. A. T. M. Phillips, P. Pankaj, C. R. Howie, A. S. Usmani, and A. H. R. W. Simpson, "Finite element modelling of the pelvis: Inclusion of muscular and ligamentous boundary conditions," Med. Eng. Phys., vol. 29, no. 7, pp. 739-748, Sep. 2007.

31. Y. Zhou, L. Min, Y. Liu, R. Shi, W. Zhang, H. Zhang, H. Duan, and C. Tu, "Finite element analysis of the pelvis after modular hemipelvic endoprosthesis reconstruction," Int. Orthop., vol. 37, no. 4, pp. 653-658, Jan. 2013.

32. N. Kaku, H. Tsumura, H. Taira, T. Sawatari, and T. Torisu, "Biomechanical study of load transfer of the pubic ramus due to pelvic inclination after hip joint surgery using a three-dimensional finite element model," J. Orthop. Sci., vol. 9, no. 3, pp. 264-269, May 2004.

33. F. Bachtar, X. Chen, and T. Hisada, "Finite element contact analysis of the hip joint," Med Biol. Eng. Comput., vol. 44, no. 8, pp. 643-651, Aug. 2006.

34. G. Bergmann, G. Deuretzbacher, M. Heller, F. Graichen, A. Rohlmann, J. Strauss, and G. Duda, Eds., "Hip contact forces and gait patterns from routine activities," 2001.

35. S. Bauer, P. Schmuki, K. von der Mark, and J. Park, "Engineering biocompatible implant surfaces," Prog. Mater. Sci., vol. 58, no. 3, pp. 261-326, Apr. 2013.

36. L. Pazos, P. Corengia, and H. Svoboda, "Effect of surface treatments on the fatigue life of titanium for biomedical applications," J. Mech. Behav. Biomed. Mater., vol. 3, no. 6, pp. 416424, Aug. 2010

37. I. P. Semenova, G. K. Salimgareeva, V. V. Latysh, T. Lowe, and R. Z. Valiev, "Enhanced fatigue strength of commercially pure Ti processed by severe plastic deformation," Mater. Sci. Eng. A, vol. 503, no. 1-2, pp. 92-95, Mar. 2009

38. R. B. Figueiredo, E. R. de C. Barbosa, X. Zhao, X. Yang, X. Liu, P. R. Cetlin, and T. G. Lang don, "Improving the fatigue behavior of dental implants through processing commercial purity titanium by equal-channel angular pressing," Mater. Sci. Eng. A, vol. 619, pp. 312-318, Dec. 2014 